\title{
The Role of the Posterolateral Tibial Slope in the Rotational Instability of the Knee in Patients Affected by a Complete Isolated Anterior Cruciate Ligament Injury: Its Value in the Decision-Making Process during the Anterolateral Ligament Reconstruction
} \author{
Mario Formagnana ${ }^{5}$ Enrico Zero ${ }^{6}$ Giacomo Zanon ${ }^{2}$ \\ ${ }^{1}$ Joint Replacement Department, IRCCS Istituto Ortopedico Galeazzi, \\ Milan, Italy \\ ${ }^{2}$ Orthopedic and Sport Traumatology Department, IRCCS Fondazione \\ Policlinico S. Matteo, Pavia, Italy \\ ${ }^{3}$ Orthopedic and Sport Traumatology Department, Ziejenhuis Oost- \\ Limburg Hospital, Genk, Belgium \\ ${ }^{4}$ Radiology and Neuroradiology Department, IRCCS Fondazione \\ Policlinico S. Matteo, Pavia, Italy \\ ${ }^{5}$ Ospedale Civile Edoardo Agnelli, Pinerolo, Italy \\ ${ }^{6}$ Department of Informatics, Bioengineering, Robotics and System \\ Engineering of the University of Genoa, Genoa, Italy
}

Marco Bargagliotti ${ }^{1}$ Francesco Benazzo ${ }^{2}$ Johan Bellemans ${ }^{3}$ Jan Truijen ${ }^{3}$ Luigi Pietrobono ${ }^{4}$

Address for correspondence Marco Bargagliotti, MD, Department of Joint Replacement, IRCCS Galeazzi Orthopaedic Institute, Via Galeazzi 4, 20161 Milan, Italy (e-mail: marcobarga@libero.it).

Joints 2019;7:78-83.

\author{
Abstract \\ Keywords \\ - anterior cruciate \\ ligament \\ - tibial slope \\ - pivot shift \\ - rotational instability \\ - posterior femoral \\ shift
}

Purpose The aim of this retrospective, multicenter study was to investigate the correlation between a high degree of rotatory instability, posterolateral tibial slope (PLTS), and anterolateral ligament (ALL) injury.

Methods The study population consisted of 76 adults with isolated, complete noncontact anterior cruciate ligament (ACL) tear. The sample was divided into two groups according to the preoperative degree of rotator instability (group A: pivot-shift test grades 2 and 3; group B: pivot-shift test grade 1). Preoperative magnetic resonance imaging (MRI) assessment included angle of PLTS, posterior shift of the lateral femoral condyle $(16 \mathrm{~mm})$ on the tibial plateau, and the presence/absence of ALL injury. The two groups were compared for differences.

Results There was a statistically significant association between pivot-shift test grades 2 and 3 (group A), PLTS slope angle $>9$ degrees, and ALL injury $(p<0.05)$. Group A also demonstrated a greater posterior shift of lateral femoral condyle $(>11 \mathrm{~mm})$, which was, however, not statistically significant when evaluated as an isolated variable.

Conclusion Our study indicates that an increased PLTS is associated with an increased incidence of ALL injury and an increased grade of pivot shift in patients with $A C L$ tear. Assessment of posterolateral tibial slope on MRI can therefore play a key adjunct role in the surgical planning of ALL reconstruction, especially in cases when ALL damage is radiologically difficult to detect or doubtful.

Level of Evidence This is a retrospective comparative level III study. received

March 4, 2019

accepted

April 4, 2020

published online

May 18, 2020
DOI https://doi.org/ $10.1055 / \mathrm{s}-0040-1710386$ ISSN 2282-4324.

\footnotetext{
(c) 2020. The Author(s).

This is an open access article published by Thieme under the terms of the Creative Commons Attribution-NonDerivative-NonCommercial-License, permitting copying and reproduction so long as the original work is given appropriate credit. Contents may not be used for commercial purposes, or adapted, remixed, transformed or built upon. (https://creativecommons.org/ licenses/by-nc-nd/4.0/) Georg Thieme Verlag KG, Rüdigerstraße 14, 70469 Stuttgart, Germany
} 


\section{Introduction}

Anterior cruciate ligament (ACL) injury poses one of the most challenging problems in knee orthopaedics. According to the Swedish National ACL Register, the incidence is approximately 50 to 80 cases per 100,000 inhabitants. $^{1-3}$

Despite advances in joint biomechanics and arthroscopic techniques, there remains much area for improvement, particularly in treating postoperative rotatory instability. ${ }^{2,4-7}$ In some cases, knee rotatory instability can persist irrespective of the type of reconstruction technique, graft choice, and coexisting injuries (e.g., meniscus or peripheral ligament tears, load-bearing axis deformation). The cause may be a poor understanding of the biomechanics of the lateral compartment of the knee. ${ }^{8,9}$ Defined by LaPrade and Bellemans as "the dark side of the knee," 10 the lateral compartment is essential for ensuring rotatory stability during ambulation, particularly in ACL-deficient knees, and suggests its importance when ligament reconstruction of the central pivot fails, ${ }^{11,12}$ resulting in poor functional outcome after surgery. ${ }^{13}$

Neoligament failure occurs more often than some may think: Sonnery-Cottet et al estimated an incidence of more than $15 \%$ in all ACL reconstructions (i.e., $6 \%$ within the first 3 years postoperative). ${ }^{14}$ Considerable debate surrounds the factors that can potentially influence knee rotatory stability. The debate has been largely fuelled by studies on the biomechanics of the anterolateral ligament (ALL).

Though considered a new frontier in sports traumatology, the ALL was originally described by the French school and much remains to be understood about its function and anatomy. ${ }^{14-18}$ Guidance for treatment is based on the orthopaedic surgeon's experience and evidence of injury on preoperative magnetic resonance imaging (MRI), which may not always be possible to detect. ${ }^{19}$ Another factor of recent interest is the role of the posterolateral tibial slope (PLTS) in providing knee static stability. ${ }^{10,20}$ While there is ample evidence for the correlation between the degree of the PLTS angle and anteroposterior knee joint instability, which increases the risk of noncontact ACL injury, the same cannot be said for the potential correlation between the PLTS angle and rotatory instability, particularly when the instability is associated with ALL damage. ${ }^{21,22}$

The aim of this retrospective multicenter study was to determine whether a correlation exists between severe clinical rotatory instability following isolated, compete ACL tear due to noncontact trauma, and PLTS angle, degree of posterior femoral shift of lateral femoral condyle on the tibial plateau, and the presence/absence of associated ALL tear.

\section{Methods}

All procedures performed in this study were in accordance with the ethical standards of the institutional and/or national research committee and with the 1964 Declaration of Helsinki and its later amendments or comparable ethical standards. We retrospectively reviewed the charts of 611 patients operated for ACL reconstruction by two senior knee surgeons: the Head of the Department of Sports Traumatol- ogy in Pavia (Fondazione IRCCS Policlinico San Matteo, Pavia, Italy) and the Head of the Department of Knee Surgery in Genk (Ziekenhuis Oost-Limburg Hospital, Genk, Belgium) between January 2013 and June 2017.

A final sample of 76 patients was obtained, with enrollment in the two aforementioned orthopaedic departments; each patient signed an informed written consent. The crosssection was divided into two subgroups according to preoperative pivot-shift test grade performed by the two heads of the departments. ${ }^{12}$ Exclusion criteria of this enrollment were partial tear of the ACL or delayed diagnosis of tear (>6 months), ligaments injuries (medial collateral, lateral collateral, or posterior cruciate ligament [PCL] injury), bone fracture, high-grade valgus or varus knee alignment (< 5 degrees), patient age $<16$ years ld or $>41$ years, and chondral knee damage greater than grade II of the International Cartilage Repair Society (ICRS) classification. Females were excluded in this study. Inclusion criteria were ACL injury after noncontact trauma occurred maximum 6 months before surgery, MRI (1.5 Tesla) findings of the isolate ACL rupture, and clinical assessment (pivot-shift test grade) reported on the outpatient chart at the diagnosis of ACL injury concordant with assessment after the patient had received spinal anesthesia (also reported on the surgical report). With this procedure, we subdivided the study sample into two groups: group $\mathrm{A}$ ( $n=38$ patients; mean age: 28.2 years), with a pivot-shift test grade between 2 and 3 (confirmed on the two orthopaedic examinations), and group $B$ ( $n=38$ patients; mean age: 29.5 years), with a pivot-shift test grade of 1 . The right knee was affected in 49 patients ( 23 in group A and 26 in group B) and the left knee in 27 (15 in group A and 12 in group B). No professional athletes were included in the study sample, and none of the patients had a sedentary lifestyle. The functional demands on the knee joint were fairly similar; all patients expected that they would be able to return to their pretreatment function level. Evaluation also included preoperative 1.5-Tesla MRI assessment (3$\mathrm{mm}$ slice thickness). Since this is a multicenter study, MRI procedures were performed with the knee flexion angle of 15 degrees. Coronal T2-weighted fat-suppressed or proton density images were acquired to identify the ALL. The medical examiner (M. B.) was assisted by a radiologist (L. P.) specialized in sports-related knee injuries. The images were evaluated using Carestream Vue PACS (Picture Archive Communication System) version 11.3.4 (Carestream Health, Rochester, New York, United States). This software has an accuracy of $0.1 \mathrm{~mm}$ and 0.1 degrees.

\section{Calculation of the Posterolateral Tibial Slope and the Posterior Femoral Shift}

The PLTS angle was calculated as described by Hudek et $a l,{ }^{22}$ according to which the preoperative MRI analysis was performed in three consecutive steps. First, the sagittal image of the knee is chosen, in which the tibial attachment of the PCL, the intercondylar eminence, and the anterior and the posterior cortices appear concave (-Fig. 1A).

Using navigational software, two circles are then traced, one cranial and the other caudal in the tibial head, so that each 

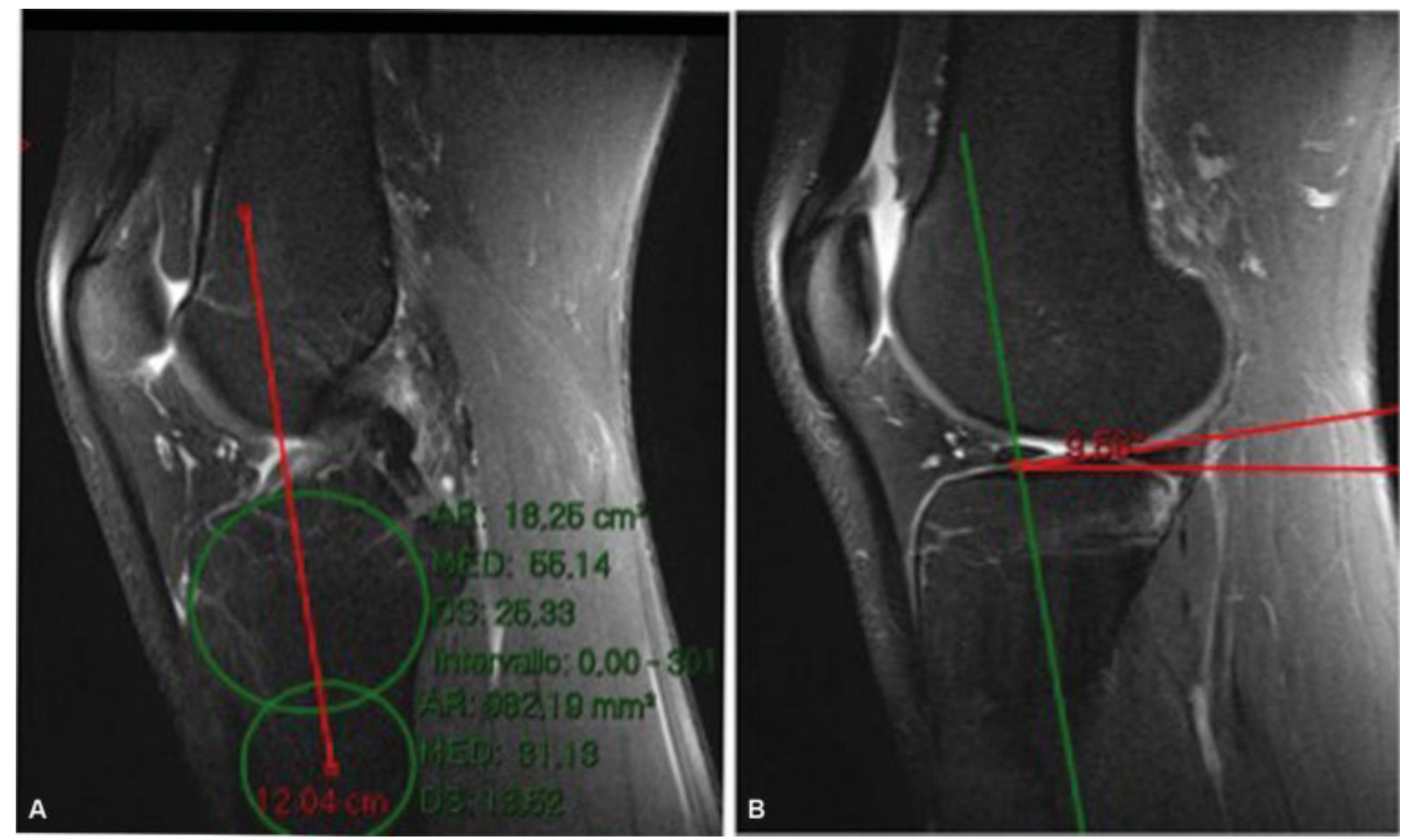

Fig. 1 The sagittal image of the knee used to calculate the longitudinal tibial axis (A) and the postero-lateral tibial slope (B) following the Hudek's protocol.

circle touches the internal cortical edges (anterior and posterior). The longitudinal tibial axis is then defined by a line that connects the centers of the two circles. With the aid of imaging software, the mediolateral center of the medial and the lateral tibial plateau is identified. The longitudinal tibial axis is then drawn at this level, and the slope angles are measured for the external and the internal plateau. The first angle is defined by the perpendicular to the longitudinal tibial axis with the tangent to the lateral tibial cortical bone surface ( - Fig. 1B). The same procedure was performed for measuring the medial plateau; in this case, the bony references were the more anterior and more posterior cortical apices of the internal tibial plateau. As suggested by Hudek et al, scrupulous attention was paid to measuring the plane passing through the cortical surface, disregarding the profile modified by the cartilage lining to avert measurement errors. The protocol described by Stoller, ${ }^{23}$ with slight modifications, was applied to calculate the posterior femoral shift. In the original method, the posterior shift of the femoral condyle is measured with respect to the medial tibial plateau. We used the external tibial plateau since rotatory stability during extension-flexion occurs mainly at that level. ${ }^{9,11}$ In this way, the posterior femoral shift on the tibia was calculated by measuring the line connecting the tangent of the posterior margin of the external tibial plateau with that of the posterior margin of the lateral femoral condyle, both of which run parallel to the longitudinal tibial axis (-Fig. 2).

\section{Data Analysis}

Continuous variables are expressed as the mean with standard deviation (SD) and were compared using Student's $t$ -

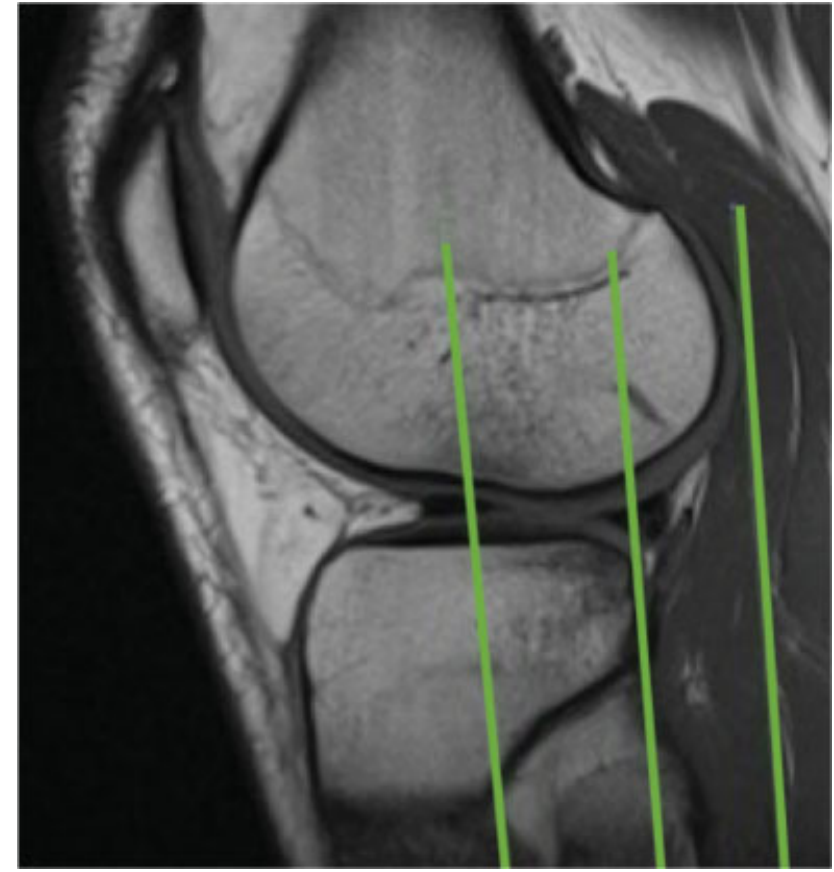

Fig. 2 The posterior femoral shift of the lateral condyle of the femur over the lateral side of the tibial plateau with subluxation of the posterior horn of the lateral meniscus.

test. The mean difference in percentage was calculated with a 95\% confidence interval. Statistical analyses were performed using Microsoft Excel (Microsoft, Redmond, Washington, United States) and MATLAB Statistic and Machine Learning Toolbox (MathWorks, Natick, Massachusetts, United States). Statistical significance was set at $p<0.05$ ( - Table $\mathbf{1}$ ). 
Table 1 Statistical analysis of the data

\begin{tabular}{|l|l|l|l|l|}
\hline & Coefficients & Standard error & p-Value & 95\% Cl \\
\hline Intercepts & 13.779 & 7.436 & 0.0000 & $(1.950 ; 31.097)$ \\
\hline Slope angle & -2.166 & 1.419 & 0.0006 & $(-4.955 ; 0.609)$ \\
\hline
\end{tabular}

Abbreviation: $\mathrm{Cl}$, confidence interval.

Table 2 The mean posterior slope angle of the lateral and the medial tibial plateau

\begin{tabular}{|l|l|l|l|l|l|}
\hline Groups & Value & \multicolumn{2}{|l|}{ Posterolateral slope } & \multicolumn{2}{l|}{ Posteromedial slope } \\
\cline { 3 - 6 } & & Right knee & Left knee & Right knee & Left knee \\
\hline Group A & Mean \pm SD & $8.9 \pm 3.1$ degrees & $9,1 \pm 2.7$ degrees & $6.6 \pm 2.8$ degrees & $6.7 \pm 2.7$ degrees \\
\hline Group B & Mean \pm SD & $6.3 \pm 2,6$ degrees & $6.1 \pm 2.5$ degrees & $4.1 \pm 2.4$ degrees & $4.3 \pm 2.3$ degrees \\
\hline
\end{tabular}

Abbreviation: SD, standard deviation.

\section{Results}

The mean posterior slope angle of the lateral and the medial tibial plateau was 9 degrees $(8.9 \pm 3.1$ degrees for the right knee, $9.1 \pm 2.7$ degrees for the left knee) and 6.7 degrees $(6.6 \pm 2.8$ degrees for the right knee, $6.7 \pm 2.7$ degrees for the left knee), respectively, in group $A$, and 6.2 degrees $(6.3 \pm 2.6$ degrees for the right knee, $6.1 \pm 2.5$ degrees for the left knee) and 4.2 degrees ( $4.1 \pm 2.4$ degrees for the right knee, $4.3 \pm 2.3$ degrees for the left knee), respectively, in group B. (-Table 2). A statistically significant difference in the mean PLTS angle (2.8 degrees) was observed between the two groups $(p<0.05)$ ( - Fig. 3 ).

ALL injury was detected in 25/38 (65.5\%) patients in group A; it was intact in 7 (18.5\%), and its entire course was difficult to identify in 6 (16\%) ( - Table 3 ). A slope angle of $>9$ degrees was measured in 24 patients, and in these patients, an ALL injury was detected in 20 and no ALL could be detected in 4 .

ALL injury was detected in $11 / 38$ (28.9\%) patients in group B: it was intact in 22 (58\%) patients, and its entire course was difficult to identify in 5 (13.1\%).

Overall, ALL was identified in $85.5 \%$ of cases, and in $56 \%$ $(n=36)$, it was completely injured (-Table 3 ). There was a statistically significant association between a PLTS angle of

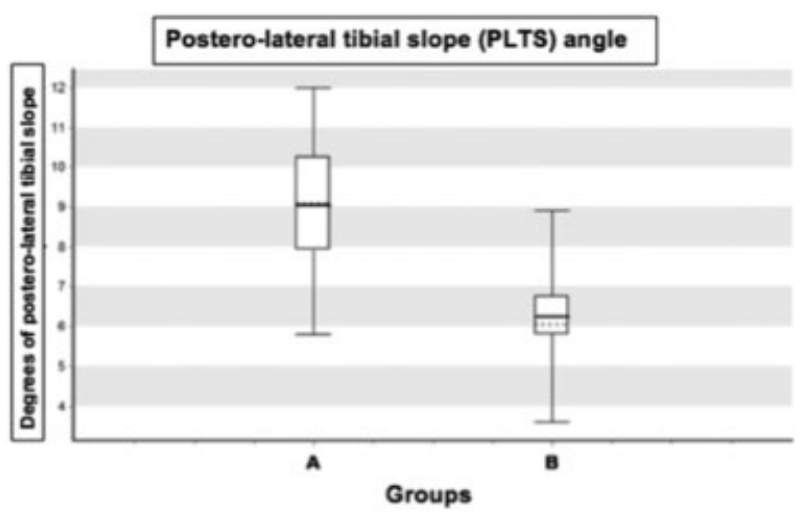

Fig. 3 Mean value of the postero-lateral tibial slope in both groups of the study. The "dashed line" represents the mean (average) value; the "continous line" represents the median value.
Table 3 MRI identification of the anterolateral ligament

\begin{tabular}{|l|l|l|l|}
\hline \multirow{2}{*}{ Groups } & \multicolumn{3}{|l|}{ Anterolateral ligament } \\
\cline { 2 - 4 } & Complete tear & No tear & Unidentified \\
\hline Group A & 25 & 7 & 6 \\
\hline Group B & 11 & 22 & 5 \\
\hline
\end{tabular}

Abbreviation: MRI, magnetic resonance imaging.

$\geq 9$ degrees in knees with isolated, complete noncontact ACL tear and ALL injury what is the $r^{2}$ value of the correlation $(p<0.05)$.

The mean posterior shift of the lateral femoral condyle on the tibial plateau was $11 \pm 5 \mathrm{~mm}$ and $6 \pm 3 \mathrm{~mm}$ in group $A$ and group $B$, respectively. We observed subluxation of the posterior lateral meniscal horn with respect to the more posterior bone angle of the tibial plateau in 66.7\% (6/9) patients with an anterior shift of $>12 \mathrm{~mm}$.

\section{Discussion}

While the contributions of soft tissue restraints, such as ligament, capsule, and meniscus, to rotatory knee laxity are relatively well understood, the contribution of bony morphology on rotatory knee laxity is less well understood. Strict inclusion criteria were applied to the study sample to minimize confounding factors. The medical charts of only 76 of 611 patients were included in the final analysis. During recruitment, approximately $21.6 \%$ of the excluded cases were noted to show discrepancies between the initial outpatient examination and the examination conducted after spinal anesthesia. One known effect of spinal anesthesia is to generate a greater knee rotatory instability due to complete relaxation of muscle tone and reduction of psychoemotive stress through induced loss of sensation, particularly painful sensations. For these 76 patients, MRI measurements were made according to the methods described by Hudek et $\mathrm{al}^{22}$ and Stoller et al, ${ }^{14}$ both of which have been demonstrated highly reliable and reproducible. But if the lateral and medial tibial compartments are often superimposed on lateral radiographic view studies (making it difficult to identify 
each plateau), MRI use allows easy measurements of isolated medial and lateral compartment of the knee.

The calculations of these parameters were performed by a single operator (M. B.) assisted by a radiologist (L. P.) specialized in sports-related knee injuries to obtain the mean of three consecutive measurements. There was no statistically significant difference in laterality (right and left knee) or age between the two groups. In line with previous observations by Hashemi et $a l,{ }^{24}$ Elmansori et al, ${ }^{20}$ Lustig et al, ${ }^{25}$ and Rahnemai-Azar et al, ${ }^{26}$ we noted a greater anteversion slope angle for the posterolateral than the posteromedial tibial plateau.

Moreover, as reported by Marouane et $\mathrm{al}^{27}$ and Brandon et al $^{28}$ the PLTS angle differed, on average, by approximately 2.8 degrees between the two groups $(p<0.05)$. This difference is consistent with evidence for an association between a PLTS angle of $\geq 12$ degrees and greater anteroposterior instability in patients with isolated, complete noncontact ACL injury. Also, Marouane et al and Brandon et al concurred that such a slope angle will also have important repercussions on rotatory stability. The cause is most likely the increased degree of freedom of tibial internal rotation with respect to the femur during flexion at 30 to 90 degrees, which can be clinically identified as a pivot-shift test grades 2 and $3 .^{12}$ In general, the medial plateau is concave and contains the medial femoral condyle during axial loading. In contrast, the lateral plateau is convex, as is the femoral condyle, which rotates over this configuration during knee motion, leading to increased mobility of the lateral compartment in uninjured knees. ACL injury weakens soft tissue restraints against anterior translation and exaggerates the mobility of the lateral compartment during knee motion. This notion, however, continues to be a subject of debate. Petrigliano et $\mathrm{al}^{29}$ demonstrated that increased tibial slope leads to increased medial compartment translation and multiplanar knee instability in PCL-deficient cadaver knees. Numerous other studies have concluded that osteotomy to modify the tibial slope angle by 5 degrees, added or subtracted, would alter rotatory stability much more than anteroposterior stability.

In line with the findings of Rahnemai-Azar et $\mathrm{al}^{26}$ our findings show that a PLTS angle of $\geq 9$ degrees is the optimal cutoff point for predicting "high-grade rotatory laxity": it is sufficient to generate rotatory instability with a high-grade pivot-shift test $(p<0.05))^{12}$ To determine whether an association exists between the PLTS and ALL injury, we compared the incidence of injury between the two groups and found that it was twice as high in group A patients. There was a statistically significant association between a PLTS angle of $\geq 9$ degrees in knees with isolated, complete ACL injury and the incidence of ALL injury $(p<0.05)$, thus confirming the clinical findings. In addition, the posterior femoral shift was greater in group A. Previous studies, including those by Klass et al in 2007, ${ }^{30}$ Tung and Davis in 1993, ${ }^{31}$ and Brandser et al in $1996,{ }^{32}$ found that a posterior femoral shift of $>7 \mathrm{~mm}$ should be considered frankly abnormal and suggestive of severe anteroposterior knee joint stability. ${ }^{30,33,34}$

We noted that a posterior femoral shift of $\geq 12 \mathrm{~mm}$, together with a PLTS angle of $>9$ degrees, strongly indicates severe rotatory instability $(p<0.05)$. Less certain is the indication in the opposite case, in which a posterior femoral shift of $\geq 12 \mathrm{~mm}$ is the only imaging abnormality (i.e., in the absence of an abnormal slope angle).

Subluxation of the posterior horn of the lateral meniscus is a hallmark sign of severe knee joint rotational and anteroposterior instability. As reported elsewhere, we found the complete course of the ALL a challenge to detect even with the use of 1.5-Tesla MRI. Cadaveric anatomical studies have reported detecting the entire ALL in 100\% of cases, whereas it has been identified by MRI in approximately $76 \%$ of cases. $^{13,19}$ Using powerful MRI machines with dedicated sequences and with the assistance of an expert radiologist, we were able to identify ALL in $85.5 \%$ of patients. Factors contributing to failure to identify ALL include previously misrecognized ALL injury due to past injury and hematoma on the lateral side of the knee at examination. The limitations of this paper include some assessments being operatordependent as the difficult to classify the real degree of clinical rotatory instability of the knee using a nonstandardized test as the pivot-shift or to take measurements with reliability using the preoperative MRI. Despite these considerations, the innovative aspect of this study is that it focuses on bone geometry with regard to static rotatory costabilizers of the knee joint. This hypothesis is supported by recent works, including that of Elmansori et al. ${ }^{20}$ This group noted also an association between greater anteversion slope of the tibial plateau, and a greater risk of ACL injury and alteration of residual rotatory instability.

Future research needs to optimize predictability of "highgrade rotatory laxity" by analyzing the role of more complex aspects of bony morphology as well as other effective factors.

This study is the first to focus on the effect of PLTS on ALL injury and the pivot-shift severity after ACL injury. Our data show that the PLTS angle indeed plays an important role in knee joint rotatory stability. Like deficiencies in other ligaments (PCL, collateral ligaments), menisci, and capsules, altered bone geometry of the tibial surface is a cofactor that potentially influences surgical outcome and risk of recurrences in patients undergoing ACL reconstruction.

Further efforts are needed to improve clinical success and prevent failures and relapses. Meticulous clinical examination and accurate preoperative MRI assessment can yield essential information that may go ignored because of lack of attention. The primary aim of scientific research in knee traumatology was to guide young orthopaedic surgeons toward evidence-medicine rather than experience-based medicine.

Conflict of Interest

None declared.

\section{References}

1 The National Scandinavian Register 2015. Available at: https:// www.aclregister.nu/media/uploads/Annual\%20reports/rapport2015en.pdf. Accessed April 29, 2020

2 Reinholdsson J, Kraus-Schmitz J, Forssblad M, Edman G, Byttner M, Stålman A. A non-response analysis of 2-year data in the 
Swedish Knee Ligament Register. Knee Surg Sports Traumatol Arthrosc 2017;25(08):2481-2487

3 Kvist J, Kartus J, Karlsson J, Forssblad M. Results from the Swedish Anterior Cruciate Ligament Register. Arthroscopy 2014;30(07): 803-810

4 Murawski CD, Wolf MR, Araki D, Muller B, Tashman S, Fu FH. Anatomic anterior cruciate ligament reconstruction: current concepts and future perspective. Cartilage 2013;4(3, Suppl):27S-37S

5 Ferreti A, Monaco E, Vadalà A. Rotatory instability of the knee after ALC tear andreconstruction. J Orthopaed TRaumatol 2014; 15:75-79

6 DePhillipo NN, Cinque ME, Chahla J, Geeslin AG, LaPrade RF. Anterolateral ligament reconstruction techniques, biomechanics, and clinical outcomes: a systematic review. Arthroscopy 2017;33 (08):1575-1583

7 Jonsson H, Riklund-Ahlström K, Lind J. Positive pivot shift after ACL reconstruction predicts later osteoarthrosis: 63 patients followed 5-9 years after surgery. Acta Orthop Scand 2004;75 (05):594-599

8 Seebacher JR, Inglis AE, Marshall JL, Warren RF. The structure of the posterolateral aspect of the knee. J Bone Joint Surg Am 1982; 64(04):536-541

9 Sanchez AR II, Sugalski MT, LaPrade RF. Anatomy and biomechanics of the lateral side of the knee. Sports Med Arthrosc Rev 2006; 14(01):2-11

10 James EW, LaPrade CM, LaPrade RF. Anatomy and biomechanics of the lateral side of the knee and surgical implications. Sports Med Arthrosc Rev 2015;23(1):2-9

11 LaPrade RF, Resig S, Wentorf F, Lewis JL. The effects of grade III posterolateral knee complex injuries on anterior cruciate ligament graft force. A biomechanical analysis. Am J Sports Med 1999; 27(04):469-475

12 Matsumoto H. Mechanism of the pivot shift. J Bone Joint Surg Br 1990;72(05):816-821

13 Slocum DB, James SL, Larson RL, Singer KM. Clinical test for anterolateral rotary instability of the knee. Clin Orthop Relat Res 1976;(118):63-69

14 Sonnery-Cottet B, Thaunat M, Freychet B, Pupim BH, Murphy CG, Claes S. Outcome of a combined anterior cruciate ligament and ante- rolateral ligament reconstruction technique with a minimum 2-year follow-up. Am J Sports Med 2015;43(07): $1598-1605$

15 Kittl C, Halewood C, Stephen JM, et al. Length change patterns in the lateral extra-articular structures of the knee and related reconstructions. Am J Sports Med 2015;43(02):354-362

16 Ingham SJM, de Carvalho RT, Martins CAQ et al. Anterolateral ligament anatomy: a comparative anatomical study. Knee Surg Sports Traumatol Arthrosc 2017;25(04):1048-1054

17 Chahla J, Menge TJ, Mitchell JJ, Dean CS, LaPrade RF. Anterolateral ligament reconstruction technique: an anatomic-based approach. Arthrosc Tech 2016;5(03):e453-e457

18 Guenther D, Griffith C, Lesniak B, et al. Anterolateral rotatory instability of the knee. Knee Surg Sports Traumatol Arthrosc 2015;23(10):2909-2917
19 Helito CP, Helito PVP, Costa HP, et al. MRI evaluation of the anterolateral ligament of the knee: assessment in routine 1.5-T scans. Skeletal Radiol 2014;43(10):1421-1427

20 Elmansori A, Lording T, Dumas R, Elmajri K, Neyret P, Lustig S. Proximal tibial bony and meniscal slopes are higher in ACL injured subjects than controls: a comparative MRI study. Knee Surg Sports Traumatol Arthrosc 2017;25(05):1598-1605

21 Jakob RP, Stäubli HU, Deland JT. Grading the pivot shift. Objective tests with implications for treatment. J Bone Joint Surg Br 1987;69 (02):294-299

22 Hudek R, Schmutz S, Regenfelder F, Fuchs B, Koch PP. Novel measurement technique of the tibial slope on conventional MRI. Clin Orthop Relat Res 2009;467(08):2066-2072

23 Stoller DW, Cannon WDJr, Anderson LJ. The knee. In: Stoller DW, ed. Magnetic Resonance Imaging in Orthopaedics and Sports Injuries. 2nd ed. Philadelphia, PA: Lippincott-Raven; 1997:203

24 Hashemi J, Chandrashekar N, Gill B, et al. The geometry of the tibial plateau and its influence on the biomechanics of the tibiofemoral joint. J Bone Joint Surg Am 2008;90(12):2724-2734

25 Lustig S, Scholes CJ, Leo SP, Coolican M, Parker DA. Influence of soft tissues on the proximal bony tibial slope measured with twodimensional MRI. Knee Surg Sports Traumatol Arthrosc 2013;21 (02):372-379

26 Rahnemai-Azar AA, Abebe ES, Johnson P, et al. Increased lateral tibial slope predicts high-grade rotatory knee laxity pre-operatively in ACL reconstruction. Knee Surg Sports Traumatol Arthrosc 2017;25(04):1170-1176

27 Marouane H, Shirazi-Adl A, Hashemi J. Quantification of the role of tibial posterior slope in knee joint mechanics and ACL force in simulated gait. J Biomech 2015;48(10):1899-1905

28 Brandon ML, Haynes PT, Bonamo JR, Flynn MI, Barrett GR, Sherman MF. The association between posterior-inferior tibial slope and anterior cruciate ligament insufficiency. Arthroscopy 2006; 22(08):894-899

29 Petrigliano FA, Suero EM, Voos JE, Pearle AD, Allen AA. The effect of proximal tibial slope on dynamic stability testing of the posterior cruciate ligament- and posterolateral corner-deficient knee. Am J Sports Med 2012;40(06):1322-1328

30 Klass D, Toms AP, Greenwood R, Hopgood P. MR imaging of acute anterior cruciate ligament injuries. Knee 2007;14(05):339-347

31 Tung GA, Davis LM. The role of magnetic resonance imaging in the evaluation of the soft tissue mass. Crit Rev Diagn Imaging 1993;34 (05):239-308

32 Brandser EA, Riley MA, Berbaum KS, el-Khoury GY, Bennett DL. MR imaging of anterior cruciate ligament injury: independent value of primary and secondary signs. AJR Am J Roentgenol 1996; 167(01):121-126

33 Tung GA, Davis LM, Wiggins ME, Fadale PD. Tears of the anterior cruciate ligament: primary and secondary signs at MR imaging. Radiology 1993;188(03):661-667

34 Brandser EA, Riley MA, Berbaum KS, el-Khoury GY, Bennett DL. MR imaging of anterior cruciate ligament injury: independent value of primary and secondary signs. AJR Am J Roentgenol 1996; 167(01):121-126 